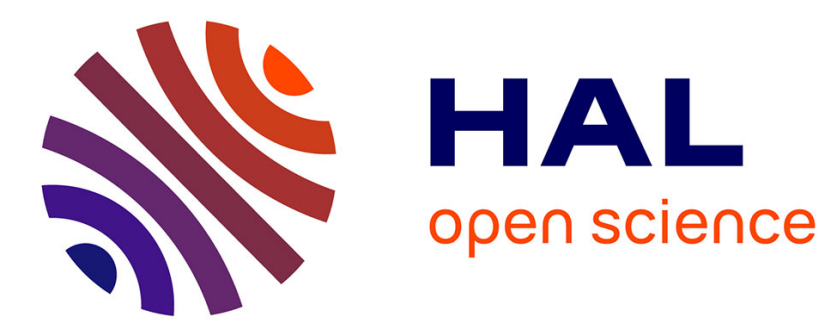

\title{
Water density and polarizability deduced from the refractive index determined by interferometric measurements up to $250 \mathrm{MPa}$
}

Laurent Weiss, Abdel Tazibt, Albert Tidu, Michel Aillerie

\section{- To cite this version:}

Laurent Weiss, Abdel Tazibt, Albert Tidu, Michel Aillerie. Water density and polarizability deduced from the refractive index determined by interferometric measurements up to $250 \mathrm{MPa}$. Journal of Chemical Physics, 2012, 136 (12), pp.124201. 10.1063/1.3698481 . hal-00699507

\section{HAL Id: hal-00699507 https://hal.science/hal-00699507}

Submitted on 21 May 2012

HAL is a multi-disciplinary open access archive for the deposit and dissemination of scientific research documents, whether they are published or not. The documents may come from teaching and research institutions in France or abroad, or from public or private research centers.
L'archive ouverte pluridisciplinaire HAL, est destinée au dépôt et à la diffusion de documents scientifiques de niveau recherche, publiés ou non, émanant des établissements d'enseignement et de recherche français ou étrangers, des laboratoires publics ou privés. 


\section{AIP $\begin{gathered}\text { mosoumalor } \\ \text { chemical Physics }\end{gathered}$}

Water density and polarizability deduced from the refractive index determined by interferometric measurements up to $250 \mathrm{MPa}$

L. Weiss, A. Tazibt, A. Tidu, and M. Aillerie

Citation: J. Chem. Phys. 136, 124201 (2012); doi: 10.1063/1.3698481

View online: http://dx.doi.org/10.1063/1.3698481

View Table of Contents: http://jcp.aip.org/resource/1/JCPSA6/v136/i12

Published by the American Institute of Physics.

Additional information on J. Chem. Phys.

Journal Homepage: http://jcp.aip.org/

Journal Information: http://jcp.aip.org/about/about_the_journal

Top downloads: http://jcp.aip.org/features/most_downloaded

Information for Authors: http://jcp.aip.org/authors

\section{ADVERTISEMENT}

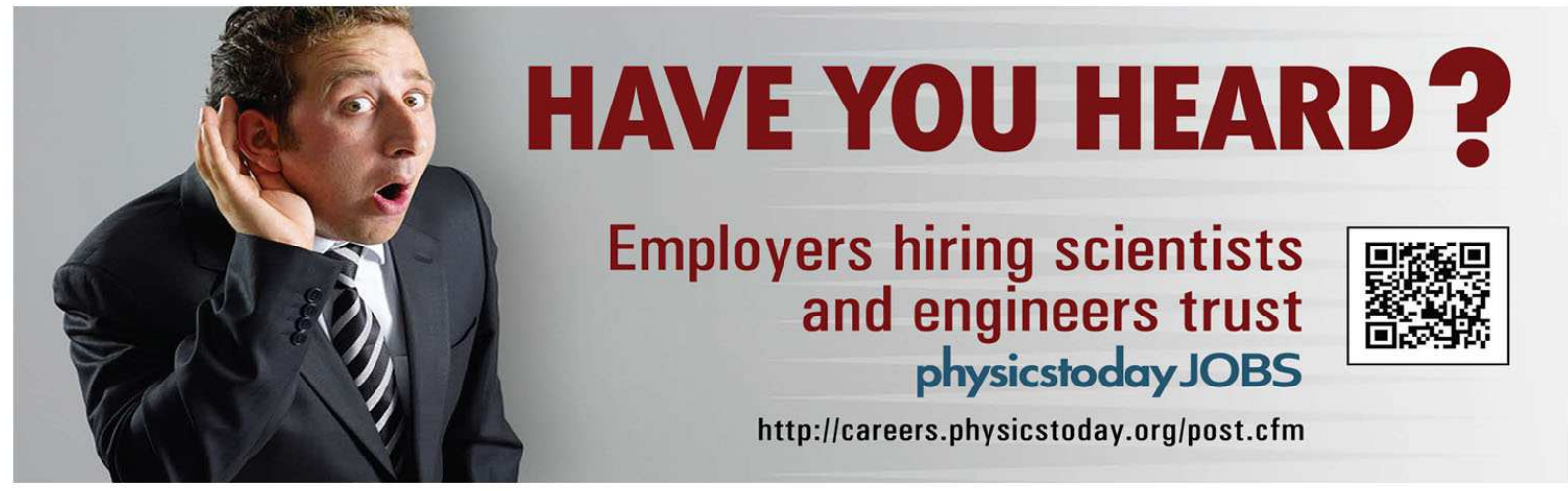




\title{
Water density and polarizability deduced from the refractive index determined by interferometric measurements up to $250 \mathrm{MPa}$
}

\author{
L. Weiss ${ }^{1,2,3}$ A. Tazibt, ${ }^{1}$ A. Tidu, ${ }^{3}$ and M. Aillerie ${ }^{2}$ \\ ${ }^{1}$ Centre de Recherche, d'Innovation et de Transfert Technologique en Jet Fluide, 2 Avenue de la Grande Terre \\ 55000 Bar-le-Duc, France \\ ${ }^{2}$ Laboratoire Matériaux Optiques, Photonique et Systèmes, Université Paul Verlaine \& Supélec, \\ 2 rue Edouard Belin, Metz, France \\ ${ }^{3}$ Laboratoire d'Etude des Microstructures et Mécanique des Matériaux, UMR CNRS 7239, \\ Université Paul Verlaine, Ile du Saulcy, Metz, France
}

(Received 22 December 2011; accepted 12 March 2012; published online 30 March 2012)

\begin{abstract}
The refractive index of water is precisely determined in the visible light range as a function of the pressure until $250 \mathrm{MPa}$ by means of a new measurement device that uses a special pipe tee included in an interferometer set. This technique allows revisiting the Bradley-Tait and Sellmeier equations to make them dependent on the wavelength and the pressure, respectively. The Bradley-Tait equation for the pressure dependence of the water refractive index is completed by a wavelength-dependent factor. Also, in the considered pressure and wavelength ranges, it is shown that the Sellmeier coefficients can be straightforwardly linked to the pressure, allowing the determination of the refractive index of water for either any wavelength or pressure. A new simple model allows the determination of the density of water as a function of the measured refractive index. Finally, the polarizability of water as function of pressure and wavelength is calculated by means of the Lorentz-Lorenz equation.

(C) 2012 American Institute of Physics. [http://dx.doi.org/10.1063/1.3698481]
\end{abstract}

\section{INTRODUCTION}

Refractive index of water has been carefully measured with many researchers for over a century. ${ }^{1-10}$ Water is the most prevalent liquid on earth. The description of the propagation, reflection, and absorption of light in water requires the knowledge of the dependence of its refractive index as a function of the wavelength under different physical (but also chemical) conditions such as pressure or temperature. Mainly, optical experiments carried out in water are often used as references for other liquids. It is also worth noticing that the refractive index itself can be used as an in situ probe of other physical parameters of the liquid such as its density or pressure. Despite the interest of scientists in the optical properties of water, traditionally the refractive index is given separately as a function of wavelength at a given pressure and temperature or as a function of pressure in the visible range.

Many studies attempt to describe the dependence of the refractive index properties of water on physical parameters such as pressure and wavelength. However, the available physical models give only a partial description of these properties. While some studies focus on the influence of pressure at specific visible wavelengths near ambient temperature, ${ }^{1-3}$ others emphasize the pressure dependence of the water refractive index for a given wavelength of the beam. The pressure range used in such experiments is moderate from atmospheric pressure up to a few hundred bars $(<1000$ bar, i.e., 100MPa). ${ }^{4-6}$ Moreover, since the relationship between the density and pressure of a liquid is well known, the value of the density is generally obtained experimentally from pressure measurements. The three principal techniques are based on the Archimedes cylinder, the metal bellows, and the oscillating U-tube. ${ }^{7,8}$

However, in many industrial processes, for example, in the case of a liquid flow as well as in water transportation, it is often necessary to determine in situ either the pressure or the density of the liquid. Among various methods mainly based on mechanical or electrical sensors, optical methods are considered as good candidates for the implementation of such sensors. This is due to the fact that optical measurements are seen to be strongly advantageous because of both non-intrusive and non-disturbing large-scale (in situ) methods.

The famous Lorentz-Lorenz (LL) equation ${ }^{9}$ links the density and the refractive index of a liquid using the polarizability. ${ }^{10}$ Thus, the LL formula describes the physical properties of water for a given polarizability.

Other simple methods based on mathematical or physical approaches exist in the literature to determine the expression linking both the water (medium) refractive index and the wavelength of the crossing beam to the liquid pressure. The physical translation of the obtained experimental results is usually made by using in one hand the Sellmeier equations to obtain the wavelength dispersion of the refractive index. On the other hand, this mathematical translation is made using the Bradley-Tait equation to give the dependence of the refractive index on the pressure.

To our knowledge, no previous work has shown the wavelength dependence of the water refractive index in a range of pressure exceeding $100 \mathrm{MPa}$. A number of applications, such as waterjet techniques for cutting hard and/or thick materials, require higher working pressures as high as $250 \mathrm{MPa}$. 
Our paper proposes a new measurement method and provides results beyond the present state of the art. Our new experimental setup includes a cell placed within one arm of a Michelson interferometer and allows determining the water refractive index as a function of both wavelength in the visible range and pressure from 1 to $250 \mathrm{MPa}$ (2500 bars).

Additionally, we used the Bradley-Tait ${ }^{11-14}$ and Sellmeier ${ }^{15}$ equations to link the water refractive index to the pressure and wavelength, respectively. We introduce pressure-dependant coefficients in the Sellmeier equation and wavelength-dependant coefficient in the Bradley-Tait equation. Finally, the density of the tested liquid (here water) is derived from the pressure measurements.

Our work addresses industrial applications by suggesting interferometry as a flexible method for in situ measurements of the refractive index of a liquid using a simple experimental setup. This may lead to a sensor for fast, non-intrusive, non-destructive, and accurate measurements of pressure and density of a static or flowing liquid.

\section{EXPERIMENTAL PROCEDURE}

We have designed and constructed a suitable highpressure liquid-cell which is introduced in a Michelson interferometer. The setup allows measuring the refractive index of liquids in the visible range as a function of the pressure which can be as high as $250 \mathrm{MPa}$. The setup is composed by two relatively independent parts. The first corresponds to the circuit of the liquid (here water) under test, the second to the optical interferometric arrangement, both parts are coupled in the liquid-cell. The setup is represented in Fig. 1. In the liquid circuit, water from the source at low pressure passes through

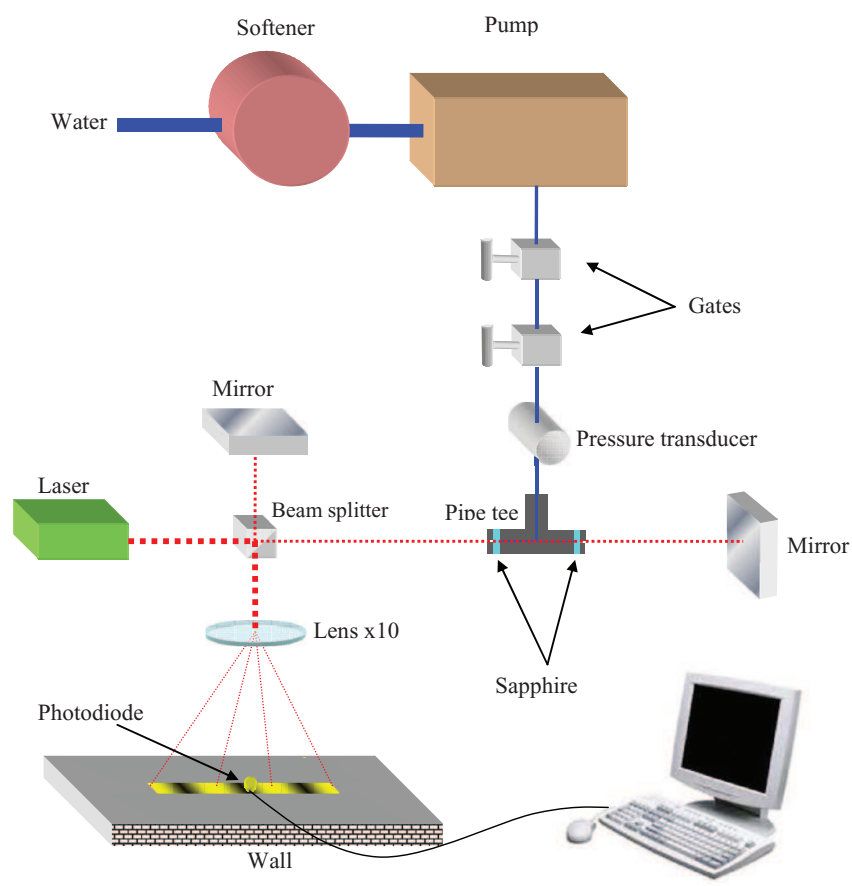

FIG. 1. The experimental setup used in these experiments. Upper part: the water circuit. Lower part: the Michelson optical setup and the computer control. a Permo 6000 softener before being put under pressure by a pump (KMT Streamline SL-IV 100D). The clean and filtered water under pressure is then channelled to the measurement system by a $1 / 4$ in. tube, interrupted by two manual security gates. After the second gate, a transducer permits the measurement of the liquid pressure in real time with an accuracy of $2 \%$.

The special liquid-cell consists of a stainless-steel body having a pipe-tee shape closed on the two opposite sides by two sapphire plano-plano windows, while the third entry is connected to the liquid circuit to complete the high-pressure flow part.

To measure the refractive index, the pipe tee is situated in the principal arm of a Michelson interferometer, with the sapphire windows perpendicular to the laser beam (Fig. 1). We have performed experiments with six different wavelengths; one emitted by a $\mathrm{Nd}: \mathrm{YVO}_{4}$ diode pump solid state laser at $\lambda=532 \mathrm{~nm}$ and the other by an adjustable He-Ne laser at $\lambda=543,594,604,612$, and $633 \mathrm{~nm}$. Other optical components of the interferometer consist in a non-polarizing beam splitter, two metallic mirrors, and a plano-convex lens ( $f=7 \mathrm{~mm}$ ) to enlarge the fringes of the interference pattern on a screen at the output of the setup. The interferometer is properly aligned so that the output fringes have a clear regular shape.

Upon variation of the liquid pressure, the interference pattern moves on the screen. The output photodiode which is located at a fixed observation point allows counting the number of scrolled fringes.

The two main experimental parameters, i.e., the pressure and the number of scrolled fringes as well as the overall control of the procedure are recorded and monitored by a computer with a LabView ${ }^{\circledR}$ homemade virtual instrument. During all the experiment, the temperature is fixed at $T=23.0 \pm 0.2^{\circ} \mathrm{C}$.

At the beginning of the experimental process, the gates are open and the water pressure is increased until $250 \mathrm{MPa}$. Then, one of the two gates is closed and the pump is powered down so that only the water after the gate in the liquid cell remains under pressure, whereas the rest of the liquid circuit empties. This procedure (pump off) prevents additional perturbations, such as mechanical vibrations in the optical part during the measurement time. At this stage, the gate is opened to let a micro water discharge allowing a slow controlled decrease in water pressure inside the cell. The optical and control measurement system is switched on and the LabView ${ }^{\circledR}$ program records the number of fringes as a function of the pressure. The setup allows getting linear pressure variation from $250 \mathrm{MPa}$ down to atmospheric pressure.

The change in the refractive index of the liquid is evaluated through the formula presented below in Eq. (1). This empiric equation is applicable to interferometric measurements only if the influence of the cell is negligible, i.e., if the shape of the cell remains quite constant when the pressure increases to $250 \mathrm{MPa}$.

At this stage, we have verified under high pressure the resistance of the components and the influence of the cell shape variation on the optical measurements. The first precaution consists in oversizing the thicknesses of both the cell 
walls and the sapphire discs. The second precaution consists in choosing the sapphire windows diameter as small as possible just letting the beam pass through the cell.

Another checks consisted in determining the optical influence of the cell on the measurements due to a possible dilatation, expansion of the cell chamber, or sapphire deformations when the pressure increases up to $250 \mathrm{MPa}$. For this purpose, a mirror is put in contact to the left sapphire disc in Fig. 1. The fringe displacement is observed during pressure increase with the beam reflected by the additional mirror, thus sensing only the left window displacement. Since no movement of the fringes was observed, we can conclude that the geometry of the cell chamber remains constant and does not affect our measurements in the considered pressure range.

Considering the value of the refractive index of water at ambient temperature and pressure, $n_{\text {Pamb }}$, known for all wavelengths from the UV up to the infrared,${ }^{10}$ the experimental relation between the refractive index $n$ and the number $Z$ of fringes passing at a fixed point is given as ${ }^{16}$

$$
n(P, \lambda)=n_{\text {Pamb }}(\lambda)+\frac{Z(P) \lambda}{2 e},
$$

where $\lambda$ is the vacuum wavelength of the beam traversing the liquid through a thickness $e$. Equation (1) allows the calculation of the refractive index for any pressure. Note that the factor $1 / 2$ in the second term of Eq. (1) is due to the special Michelson interferometer configuration where the beam crosses the cell twice.

\section{EXPERIMENTAL RESULTS AND DISCUSSION}

Figure 2 shows the pressure dependence of the water refractive index for six wavelengths in the green/red spectral range. These data are obtained using the setup of Fig. 1, Eq. (1), and the single reference values of the refractive index at atmospheric pressure. ${ }^{9,10}$

It can be seen from Fig. 2 that the refractive index of water nonlinearly increases as a function of pressure, particularly at high pressures. This property of water, obtained from our measurements for various wavelengths, confirms the results of Vedam and Limsuwan ${ }^{1}$ obtained at a fixed single wavelength of $583 \mathrm{~nm}$. To fit our curves, and to benefit from previous published results, we consider the standard relationship $k=n$ between the dielectric constant $k$ and the refractive

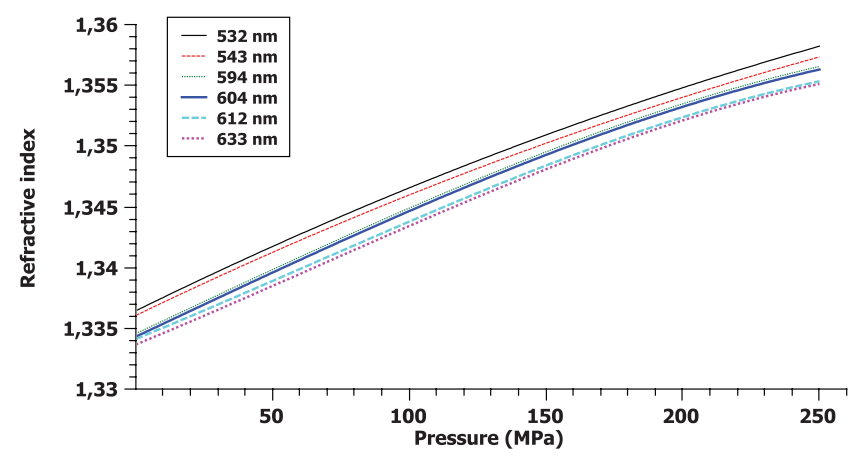

FIG. 2. Evolution of the refractive index as a function of pressure for various wavelengths in the visible range. index $n$ in a lossless medium. ${ }^{17}$ Bradley and Pitzer $^{11}$ have proposed a simple but effective description of the pressure dependence of the dielectric constant based on the equation suggested by Tait in 1880 for volumetric data. ${ }^{14}$ The BradleyPitzer equation takes the following form:

$$
k(P)=k_{\left(P=P_{i n i}\right)}+a_{0} \ln \left(\frac{a_{1}+P}{a_{1}+P_{i n i}}\right),
$$

where $k_{(P}=$ Pini $)$ is the dielectric constant at a given reference pressure $P_{i n i}, P$ is the measured pressure in $\mathrm{MPa}$ and $a_{0}$ and $a_{1}$ are two constant parameters that can be deduced by the experimental data. This equation was previously used to fit the dielectric data available from the International Association for the Properties of Water and Steam with accuracy better than $0.1 \%$ from atmospheric pressure up to some hundred $\mathrm{MPa}^{7}$

Equation (2) leads directly to the relation

$$
n=\sqrt{n_{0}^{2}(\lambda)+a_{0} \ln \left(\frac{a_{1}+P}{a_{1}+100}\right)},
$$

where $P$ and $a_{1}$ are expressed in MPa. Note that the wavelength dependence of the refractive index appears in Eq. (3) only within the term $n_{0}$ and all other parameters are considered independent from the wavelength. The best fitting parameters for Eq. (3) are

$$
\begin{aligned}
& n_{0}^{2}=n_{P_{a m b}}^{2}+3.0516 \times 10^{-3} \pm 10^{-7}, \\
& a_{0}=0.1057 \pm 10^{-4}, \\
& a_{1}=341.5902 \pm 10^{-4} \mathrm{MPa}
\end{aligned}
$$

where the term $n^{2}\left(\lambda, P_{0.1 \mathrm{MPa}}\right)$ is the refractive index value at atmospheric pressure at given wavelength.

On the other hand, using the least-square method, we can fit the experimental data obtained for the refractive index at various pressures using Sellmeier dispersion formula of the form

$$
n(P, \lambda)=1+\sum_{i=1}^{s} \frac{B_{i}(P) \lambda}{\lambda-C_{i}(P)},
$$

with $B_{i}$ and $C_{i}$ being Sellmeier dispersion coefficients considered as pressure dependent.

We have evaluated the residual deviation between the values calculated by this dispersion formula and the measurement values with various fitting orders s in the Sellmeier relationship. These standard residuals deviations are found to be smaller than $5.4 \times 10^{-4}$ in our pressure range when considering a three-oscillator Sellmeier dispersion formula. This accuracy makes it possible to approximate the experimental results to the fourth decimal digit. The accuracy obtained in the present work is about the one obtained by the BradleyPitzer model to fit the refractive index of water. The evolution of the six dispersion coefficients of the three-oscillator Sellmeier equation with $s=3$ is given in Fig. 3 .

The pressure dependence of the coefficients $B_{i}$ and $C_{i}$ in Eq. (4) can be reasonably described by a second-order 

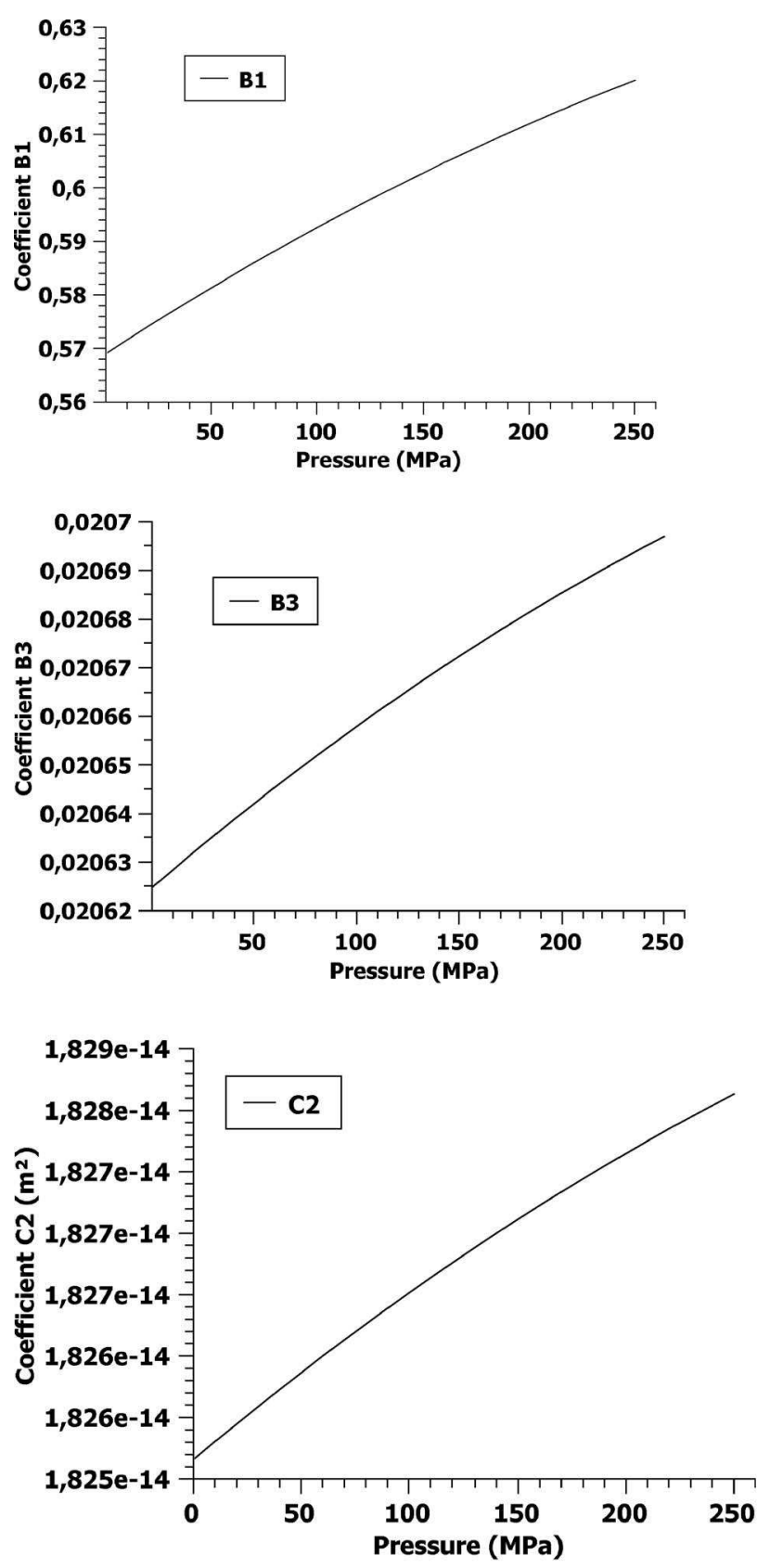
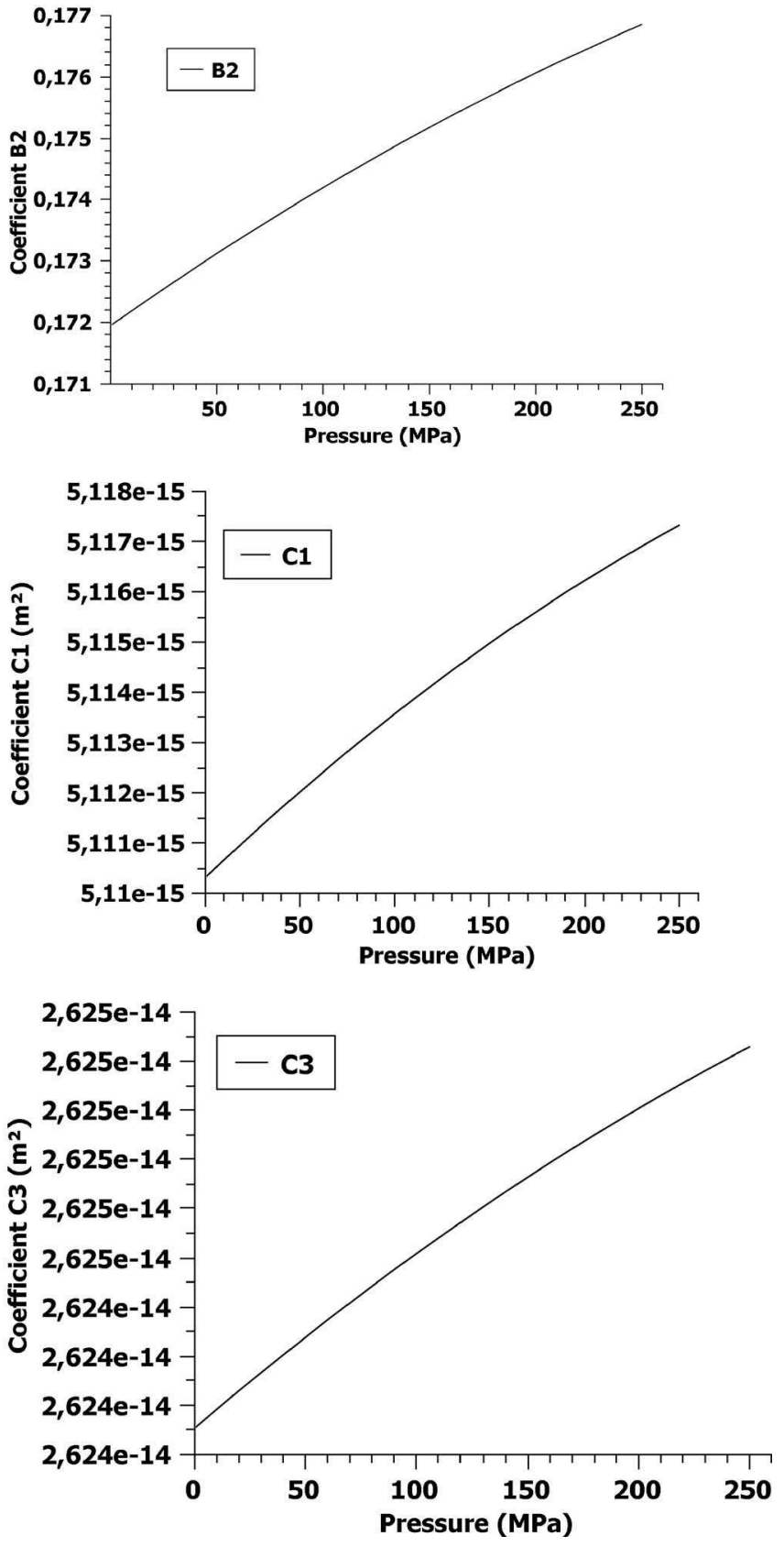

FIG. 3. Sellmeier dispersion coefficients for the refractive index of water as a function of pressure at $23^{\circ} \mathrm{C}$.

polynomial so that Eq. (4) can be rewritten as

$$
n^{2}=1+\sum_{i=1}^{3} \frac{\left(\alpha_{i} P^{2}+\beta_{i} P+\delta_{i}\right) \lambda^{2}}{\lambda^{2}-\left(\mathrm{A}_{i} P^{2}+\mathrm{B}_{i} P+\Delta_{i}\right)},
$$

where $\alpha_{i}, \beta_{i}$, and $\delta_{i}$ on the one hand and the same capital Greek letters $A_{i}, B_{i}$, and $\Delta_{i}$ on the other hand are the coefficients of the polynomials describing the $B_{i}$ and $C_{i}$ Sellmeier coefficients of Eq. (4), respectively. The coefficients of Eq. (5) are given in Table I.

\section{DISCUSSION}

We can now compare the two models described above by Bradley-Pitzer and Sellmeier. In Fig. 4, the fits of the BradleyPitzer and the Sellmeier models are shown for the two wavelengths $532 \mathrm{~nm}$ and $633 \mathrm{~nm}$. A reasonable agreement between both models and the measurements can be observed.

As the fringes in the interference pattern present a good contrast, the error in the counting process is very small. However, we estimate the maximum level of counting error during all the experimental process equal to 10 fringes lost over a total amount of around 1500 fringes passing in front of the photodiode. These experimental uncertainties can induce a change in the refractive index equal to $6.7 \times 10^{-5}$ and 7.9 
TABLE I. Polynomial coefficients for the generalized Sellmeier relationship (Eq. (5)) for the refractive index of water at the temperature of $23{ }^{\circ} \mathrm{C}$ (see Eq. (5)).

\begin{tabular}{lcccccc}
\hline \hline & $\begin{array}{c}\alpha \\
\left(\mathrm{MPa}^{-2}\right)\end{array}$ & $\begin{array}{c}\beta \\
\left(\mathrm{MPa}^{-1}\right)\end{array}$ & $\delta$ & $\begin{array}{c}\mathrm{A} \\
\left(\mathrm{m}^{2} \mathrm{MPa}^{-2}\right)\end{array}$ & $\begin{array}{c}\mathrm{B} \\
\left(\mathrm{m}^{2} \mathrm{MPa}^{-1}\right)\end{array}$ \\
\hline 1 & $-2.08621659 \times 10^{-11}$ & $2.56337533 \times 10^{-6}$ & $5.69053109 \times 10^{-1}$ & $-3.05408679 \times 10^{-9}$ & $3.56454006 \times 10^{-4}$ & $5.11031710 \times 10^{3}$ \\
2 & $-1.90689751 \times 10^{-12}$ & $2.43746527 \times 10^{-7}$ & $1.71949519 \times 10^{-1}$ & $-1.13105732 \times 10^{-8}$ & $1.47164417 \times 10^{-3}$ & $1.82515802 \times 10^{4}$ \\
3 & $-2.83966194 \times 10^{-14}$ & $3.59587056 \times 10^{-9}$ & $2.06248215 \times 10^{-2}$ & $-2.96996177 \times 10^{-9}$ & $3.84125453 \times 10^{-4}$ & $2.62415393 \times 10^{4}$ \\
\hline \hline
\end{tabular}

$\times 10^{-5}$ for $532 \mathrm{~nm}$ and $633 \mathrm{~nm}$, respectively. The experimental accuracy expected in the determination of the refractive index of water by our interferometric setup is thus better than $0.006 \%$. The modelling of the refractive index versus pressure through Eq. (3) or Eq. (4) induces new errors. These errors can be estimated by determining the discrepancy between experimental and fitted curves found equal to $0.02 \%$ for the Bradley-Pitzer approach and $0.04 \%$ for the Sellmeier one. Therefore, experimental uncertainties are about 10 times smaller than the errors induced by the fitting models. Finally, the overall uncertainties in the determination of the refractive index of water equal $0.05 \%$ by using the Sellmeier formula (Eq. (5)) and $0.03 \%$ by using the Bradley-Pitzer formula (Eq. (3)).

Thus, we can conclude that the two models are well adapted for the description of the room temperature refractive index of water as a function of the pressure in the light visible range. It would have been interesting to compare these two models also outside the visible spectrum. However, the extrapolated accuracy of our relationships in the ultraviolet (UV) and infrared (IR) ranges as are expected to be less good. On one hand, our measurements were done only in the visible range. On the other hand, earlier works on the water refractive index outside the visible range show generally an increased discrepancy between the fits and the refractive index measurements. However, the precise description of the water refractive index in the UV or IR ranges is outside the scope of the present work.

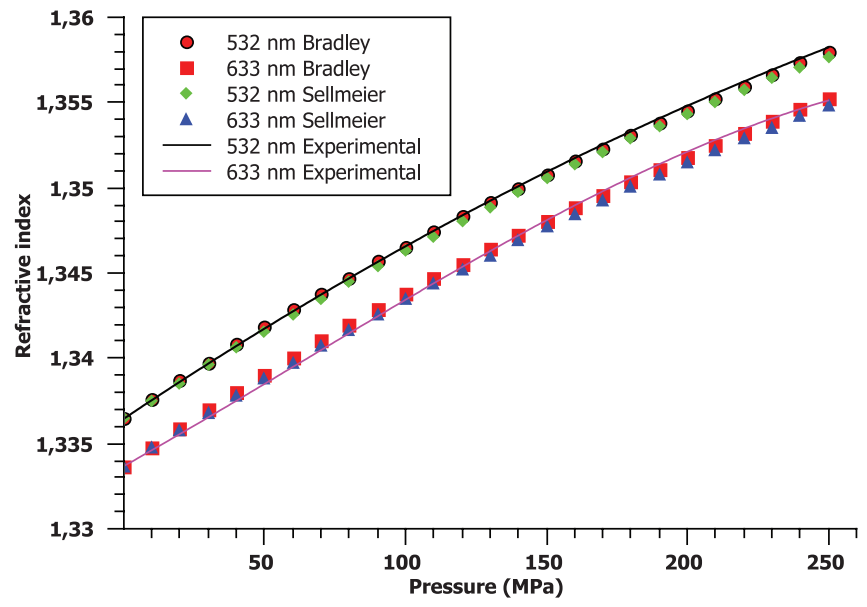

FIG. 4. Comparison between our experimental data of the refractive index of water and data provided by the Bradley-Pitzer and the Sellmeier dispersion formula.
To define the relationship between the density and the refractive index of water at different wavelengths, we first link the density to the pressure using the Tait equation in its modified form (Eq. (6)). It is worth noting that the original Tait equation in its inverted form gives an excellent representation of density data for liquids at pressures up to only $15 \mathrm{MPa} .^{12}$ For density measurements of liquids at higher pressures, it is better to use the modified Tait equation that greatly improves the fits. ${ }^{18}$ The modified Tait equation for the density $\rho$ of water is ${ }^{7}$

$$
\rho=\rho_{(P=P i n i)}+a_{2} \ln \left(\frac{a_{3}+P}{a_{3}+P_{\text {ini }}}\right) .
$$

In Ref. 7, Floriano and Nascimento determined precisely the coefficient values $a_{2}$ and $a_{3}$. The density values calculated using the proposed method matches well those given in standard tables. ${ }^{19,20}$ Using Eq. (6) to relate density and pressure, and Eq. (5) or Eq. (3) to relate pressure and refractive index, one can determine the liquid density by a refractive index measurement. Therefore, the density of a static or flowing liquid can be determined with the help of a laser and an interferometric setup. This is done in Fig. 5, where we have calculated the water density from the values of its refractive index for various wavelengths using Eqs. (3) and (6). We can observe that for a given density, the refractive index decreases with the wavelength of the probe laser beam. It is clear that the interferometric setup and the experimental method

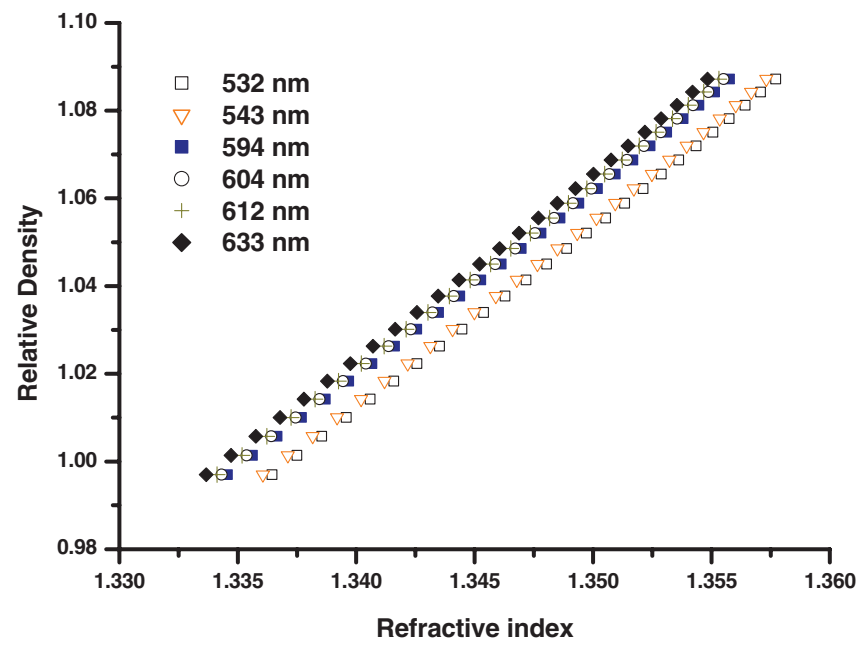

FIG. 5. Relative density of water as a function of refractive index for different wavelengths at $23^{\circ} \mathrm{C}$. 

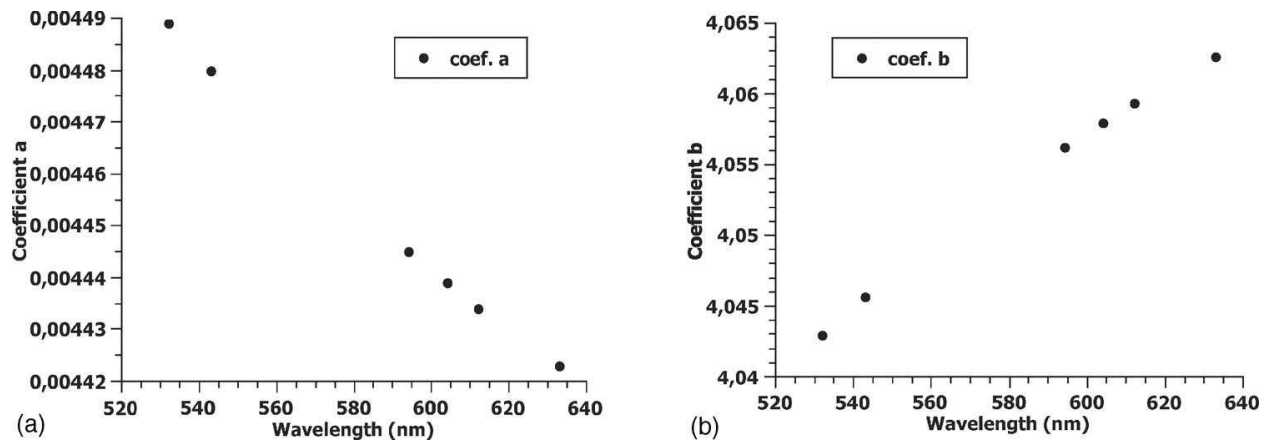

FIG. 6. Coefficients (a) and (b) empirically relating the relative density and the refractive index (Eq. (7)) as a function of wavelength.

constitute a non-intrusive, non-destructive sensor for both pressure and density measurements.

We have modelled the relationship between the density and the refractive index of water at room temperature. For that purpose, we have fitted the curves of Fig. 5 with various polynomials and other nonlinear equations. The best fits of our experimental data of the relative density for all curves are obtained with an exponential function of the refractive index $n$. The basic formula used is

$$
d(\lambda, n)=a(\lambda) e^{b(\lambda) n}
$$

with $a$ and $b$ defined for each wavelength.

By fitting our data through Eq. (7) for each wavelength, we obtain the two coefficients $a$ and $b$. Their behaviour as a function of the wavelength is plotted in Fig. 6. The graphs show that the evolution of these two coefficients follows a nonlinear dependence with the wavelength and this dependence can be described by a second-degree polynomial equation. As a consequence, the generalisation of Eq. (7) for the visible spectra range used in this study gives the density as follows:

$$
d(\lambda, n)=\left(a_{2} \lambda^{2}+a_{1} \lambda+a_{0}\right) e^{\left(b_{2} \lambda^{2}+b_{1} \lambda+b_{0}\right) n} .
$$

The coefficients of Eq. (8) are given in Table II.

With these coefficients, the maximal discrepancy among the model of Eq. (8), the experimental values of the refractive index, and the values of the relative density is equal to $0.19 \%$.

Thanks to Eqs. (8), (3), and (5), and thanks to the coefficients of Table II, it becomes easy to determine the density of static or flowing water using interferometry measurements. We want to underline some specific advantages of this method. The first advantage is that the density of the water can be obtained without the knowledge of the water pressure, as usually the case in industrial process. The second advantage is the high accuracy in the determination of the density changes expected with the proposed method. As we have further noticed, from a fix position of the fringe pattern at the output of the interferometric setup and at a given pressure (density), a change in the pattern observed by the photodiode is directly associated with a pressure (density) change. According to intrinsic characteristics of our experimental device, the difference between two bright (or two dark) fringes corresponds to a change of the relative density value, $\Delta d$ equal to $6 \times 10^{-5}$. Finally, another advantage is that the proposed method can be applied to determine in situ the pressure and the density of various liquids, such as mediums in petrochemistry, chemistry, and others liquids.

Based on a quite simple mathematical approach, the previous formulas of our model directly provide the link among refractive index, relative density, pressure, and wavelength. Nevertheless and as previously mentioned in the Introduction, the physical Lorentz-Lorenz formula introduces the concept of polarizability noted $\alpha$. The normal form of the LL formula can be written as

$$
\frac{n^{2}-1}{n^{2}+2}=\frac{4 \pi d N_{A}}{3 M} \alpha
$$

with $n$ the refractive index, $d$ the density, $N_{A}$ the Avogadro constant, and $M$ the molar mass.

Thanks to our refractive index measures and the density values, we can plot (see Fig. 7) the polarizability of water as function of pressure for various wavelengths.

From the characteristics plotted in Fig. 7, one can directly obtain the porizability values of water under pressure up to $250 \mathrm{MPa}$. The polarizability values as a function of density and pressure at every $10 \mathrm{MPa}$ are reported in Table III.

The LL formula is mainly valid for pure organic liquid. ${ }^{21}$ To improve the LL formula by another function of $n$,

TABLE II. Coefficients of the Eq. (8) related to the relative density behaviour as a function of the refractive index of water at $23^{\circ} \mathrm{C}$ in the visible spectra range.

\begin{tabular}{lcccc}
\hline \hline $\begin{array}{l}a_{2} \\
\left(\mathrm{~m}^{-2}\right)\end{array}$ & $\begin{array}{c}a_{1} \\
\left(\mathrm{~m}^{-1}\right)\end{array}$ & $a_{0}$ & $\begin{array}{c}b_{2} \\
\left(\mathrm{~m}^{-2}\right)\end{array}$ & $\begin{array}{c}b_{1} \\
\left(\mathrm{~m}^{-1}\right)\end{array}$ \\
\hline $1.45781289 \times 10^{-9}$ & $-2.35046033 \times 10^{-6}$ & $5.32669029 \times 10^{-3}$ & $-4.85379246 \times 10^{-7}$ & $7.59564314 \times 10^{-4}$ \\
\hline \hline
\end{tabular}


TABLE III. Polarizability calculated with Lorentz-Lorenz formula as function of density and pressure from 1 to $250 \mathrm{MPa}$ for various wavelengths in the visible range at $23^{\circ} \mathrm{C}$.

\begin{tabular}{|c|c|c|c|c|c|c|c|}
\hline \multirow[b]{2}{*}{ Relative density (Ref. 19) } & \multirow[b]{2}{*}{ Pressure (Mpa) } & \multicolumn{6}{|c|}{ Polarizability $\left(10^{-30} \mathrm{~m}^{3}\right)$} \\
\hline & & $532 \mathrm{~nm}$ & $543 \mathrm{~nm}$ & $594 \mathrm{~nm}$ & $604 \mathrm{~nm}$ & $612 \mathrm{~nm}$ & $633 \mathrm{~nm}$ \\
\hline 0.9975 & 1 & 1.4864 & 1.4849 & 1.4789 & 1.4779 & 1.4772 & 1.4753 \\
\hline 1.0014 & 10 & 1.4849 & 1.4834 & 1.4774 & 1.4764 & 1.4757 & 1.4738 \\
\hline 1.0058 & 20 & 1.4827 & 1.4811 & 1.4752 & 1.4742 & 1.4734 & 1.4716 \\
\hline 1.0100 & 30 & 1.4805 & 1.4790 & 1.4730 & 1.4720 & 1.4713 & 1.4694 \\
\hline 1.0142 & 40 & 1.4783 & 1.4768 & 1.4709 & 1.4699 & 1.4692 & 1.4673 \\
\hline 1.0183 & 50 & 1.4763 & 1.4748 & 1.4689 & 1.4679 & 1.4671 & 1.4653 \\
\hline 1.0223 & 60 & 1.4743 & 1.4728 & 1.4669 & 1.4659 & 1.4651 & 1.4633 \\
\hline 1.0263 & 70 & 1.4723 & 1.4708 & 1.4649 & 1.4640 & 1.4632 & 1.4614 \\
\hline 1.0302 & 80 & 1.4704 & 1.4689 & 1.4630 & 1.4621 & 1.4613 & 1.4595 \\
\hline 1.0340 & 90 & 1.4685 & 1.4670 & 1.4612 & 1.4602 & 1.4595 & 1.4577 \\
\hline 1.0377 & 100 & 1.4667 & 1.4652 & 1.4594 & 1.4584 & 1.4577 & 1.4559 \\
\hline 1.0414 & 110 & 1.4649 & 1.4634 & 1.4576 & 1.4566 & 1.4559 & 1.4541 \\
\hline 1.0450 & 120 & 1.4631 & 1.4616 & 1.4558 & 1.4549 & 1.4541 & 1.4523 \\
\hline 1.0486 & 130 & 1.4613 & 1.4599 & 1.4541 & 1.4531 & 1.4524 & 1.4506 \\
\hline 1.0521 & 140 & 1.4596 & 1.4581 & 1.4524 & 1.4514 & 1.4507 & 1.4489 \\
\hline 1.0555 & 150 & 1.4579 & 1.4564 & 1.4507 & 1.4497 & 1.4490 & 1.4472 \\
\hline 1.0589 & 160 & 1.4562 & 1.4548 & 1.4490 & 1.4481 & 1.4473 & 1.4456 \\
\hline 1.0622 & 170 & 1.4546 & 1.4531 & 1.4474 & 1.4464 & 1.4457 & 1.4439 \\
\hline 1.0655 & 180 & 1.4529 & 1.4514 & 1.4457 & 1.4448 & 1.4441 & 1.4423 \\
\hline 1.0687 & 190 & 1.4513 & 1.4498 & 1.4441 & 1.4432 & 1.4424 & 1.4407 \\
\hline 1.0719 & 200 & 1.4496 & 1.4482 & 1.4425 & 1.4415 & 1.4408 & 1.4391 \\
\hline 1.0751 & 210 & 1.4480 & 1.4465 & 1.4409 & 1.4399 & 1.4392 & 1.4374 \\
\hline 1.0782 & 220 & 1.4464 & 1.4449 & 1.4392 & 1.4383 & 1.4376 & 1.4358 \\
\hline 1.0812 & 230 & 1.4447 & 1.4433 & 1.4376 & 1.4367 & 1.4360 & 1.4342 \\
\hline 1.0842 & 240 & 1.4431 & 1.4417 & 1.4360 & 1.4351 & 1.4344 & 1.4326 \\
\hline 1.0872 & 250 & 1.4415 & 1.4401 & 1.4344 & 1.4335 & 1.4328 & 1.4310 \\
\hline
\end{tabular}

Looyenga (LO) suggests an alternate equation ${ }^{22}$

$$
n^{2 / 3}-1=\frac{4 \pi d N_{A}}{3 M} \alpha .
$$

The difference between the LL and LO formulas exists only in the third term of the expansion of the

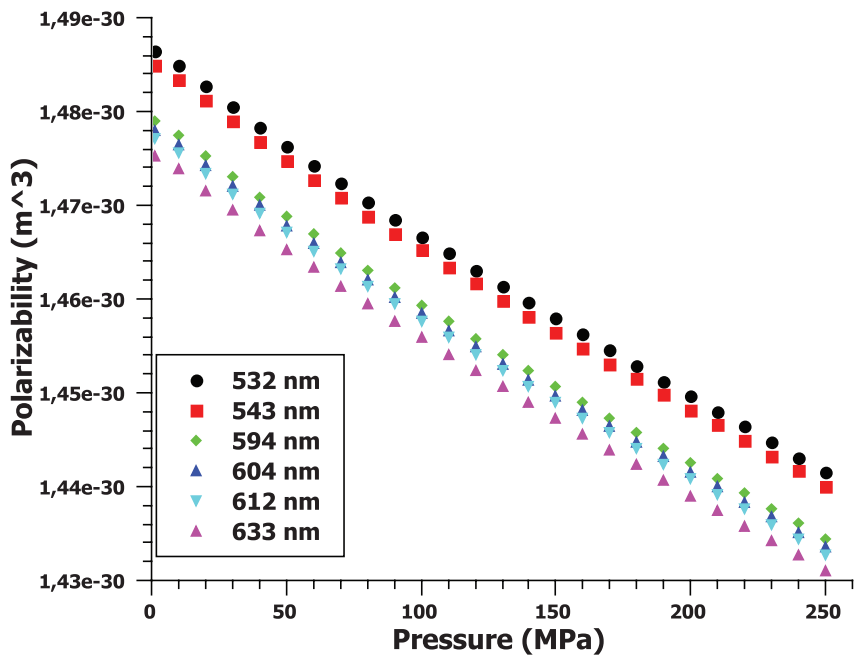

FIG. 7. Polarizability of water as a function of pressure for various wavelengths at $23^{\circ} \mathrm{C}$.
Eqs. (9) and (10) in $n-1 .{ }^{23}$ In our case, the calculated polarizability is $2.7-3 \%$ higher with LO than with LL.

\section{CONCLUSION}

Using a special pipe tee within a Michelson interferometer, we have developed a simple and original device and a method for the in situ measurement of physical properties of static or flowing liquids under low-to-high pressure. We have applied the implemented method for the determination of the room temperature refractive index and the density of pure water from $250 \mathrm{MPa}$ ( 2500 bars) to the atmospheric pressure at room temperature. From the interferometric measurements, the refractive index of the liquid under test can be directly calculated. We have determined the dispersion of the refractive index of water in the visible range for pressure up to $250 \mathrm{MPa}$. The pressure is then deduced either by a modified Bradley-Pitzer or a modified Sellmeier equation, both giving satisfactory accuracy in the pressure range we have studied. Using the modified Tait equation linking density to the pressure, we have established a simple relationship between the density and the refractive index. We have shown that a simple exponential law can easily fit this relationship. In order to propose an overall method for the visible spectrum, the correlation coefficients are given as function of the wavelength. Thanks to the Lorentz-Lorenz equation and from 
experimental results, we have determined the polarizability of water at room temperature and pressure up to $250 \mathrm{MPa}$. In this paper, we have shown that the three formulas, BradleyPitzer-Tait, Sellmeier, and Lorentz-Lorenz can be considered as equivalent formulas for the determination of the refractive index of water in the considered pressure range. Nevertheless, we have shown that only the pressure is needed to obtain the refractive index with Tait or Sellmeier equations with the same accuracy and without the necessary knowledge of other physical parameters such as either the polarizability or density that are currently needed in the LL equation. Finally, the interferometric method associated with the specific proposed setup presented in this paper can be considered as an accurate nonintrusive and non-perturbing pressure-density optical sensor for static or flowing liquid measurements.

\section{ACKNOWLEDGMENTS}

The authors wish to express their great gratitude to Professor Germano Montemezzani for the fruitful scientific discussion and careful revision of the manuscript and Nicolas Delpuech and Yves Godefert for their active participation in the experiments.

${ }^{1} \mathrm{~K}$. Vedam and P. Limsuwan, "Piezo-optic behavior of water and carbon tetrachloride under high pressure," Phys. Rev. Lett. 35(15), 1014-1016 (1975).

${ }^{2}$ R. M. Waxler and C. E. Weir, "Effect of pressure and temperature on the refractive indices of benzene, carbon tetrachloride, and water," J. Res. Natl. Bur. Stand. Sec. A 67A(2), 163-171 (1963).

${ }^{3}$ A. H. Harvey, J. S. Gallagher, and J. M. H. Levelt Sengers, "Revised formulation for the refractive index of water and steam as a function of wavelength, temperature and density," J. Phys. Chem. Ref. Data 27(4), 761-774 (1998).

${ }^{4}$ D. K. Lynch and W. Livingston, Colour and Light in Nature, see Table 4.3, (Cambridge University Press, 1995) (1st ed.) and 2001 (2nd ed.), ISBN 0521775043
${ }^{5}$ D. Segelstein, "The complex refractive index of water," M. S. thesis, University of Missouri-Kansas City, 1981 (data can be downloaded as "Segelstein.txt" when downloading the MiePlot program).

${ }^{6}$ International Association for the Properties of Water and Steam, Release on Refractive Index of Ordinary Water Substance as a Function of Wavelength, Temperature and Pressure (1997).

${ }^{7}$ W. B. Floriano and M. A. C. Nascimento, "Dielectric constant and density of water as a function of pressure at constant temperature," Braz. J. Phys. 34(1), 38-41 (2004).

${ }^{8} \mathrm{P}$. W. Bridgman, "The pressure-volume-temperature relations of the liquid, and phase diagram of heavy water," J. Chem. Phys. 3(10), 597-605 (1935).

${ }^{9}$ H. Eisenberg, "Equation for the refractive index of water," J. Chem. Phys. 43(11), 3887-3892 (1965).

${ }^{10} \mathrm{P}$. Schiebener et al., "Refractive index of water and steam as function of wavelength temperature and density," J. Phys. Chem. Ref. Data 19(3), 677717 (1990).

${ }^{11}$ D. J. Bradley and K. S. Pitzer, "Thermodynamics of electrolytes. Dielectric properties of water and Debye-Hückel parameters to $350^{\circ} \mathrm{C}$ and $1 \mathrm{kbar}$," J. Phys. Chem. 83, 12 (1979).

${ }^{12}$ J. H. Dymond and R. Malhotra, "The Tait equation: 100 years on," Int. J. Thermophys. 9(6), 941-951 (1988).

${ }^{13}$ G. A. Neece and D. R. Squire, "On the Tait and relate empirical equation of state," J. Phys. Chem. 78(1), 128-136 (1968).

${ }^{14}$ P. G. Tait, Physics and Chemistry of the Voyage of H.M.S. Challenger (HMSO, London, 1888), Vol. 2, Part 4

${ }^{15}$ M. Daimon and A. Masumura, "Measurement of the refractive index of distilled water from the near-infrared region to the ultraviolet region," Appl. Opt. 46(18), 3811-3820 (2007).

${ }^{16} \mathrm{M}$. Born and E. Wolf, Principles of Optics, 7th ed. (Cambridge University Press, 1999), p. 302

${ }^{17}$ J. D. Tilley, Understanding Solids: The Science of Materials (Wiley, 2004), p. 446.

${ }^{18}$ Handbook of Chemistry and Physics, 47th ed. (Chemical Rubber, Cleveland, OH, 1966), p. F9.

${ }^{19}$ Handbook of Chemistry and Physics, 79th ed. (CRC-Press, 1999), Chap. 6.

${ }^{20} \mathrm{M}$. Tanaka et al., "Recommended table for the density of water between $0{ }^{\circ} \mathrm{C}$ and $40{ }^{\circ} \mathrm{C}$ based on recent experimental reports," Metrologia 38, 301-309 (2001).

${ }^{21} \mathrm{~F}$. Croccolo et al., "Concentration dependent refractive index of a binary mixture at high pressure," J. Chem. Phys. 135, 034901 (2011).

${ }^{22} \mathrm{H}$. Looyenga, "Dielectric constants of homogeneous mixture," Mol. Phys. 9, 501 (1965).

${ }^{23}$ W. B. Li et al., "Determination of the temperature and concentration dependence of the refractive index of a liquid mixture," J. Chem. Phys. 101, 5058 (1994). 